\title{
ON EXTREMAL PROPERTIES OF THE DERIVATIVES OF POLYNOMIALS AND RATIONAL FUNCTIONS
}

$$
\text { M. A. Malik }
$$

(received August 17, 1962)

Introduction. Let $\mathrm{p}(\mathrm{z})$ be a polynomial of degree $\mathrm{n}$, i.e. a finite sum of the form $\sum_{\nu=0}^{n} c_{\nu} z^{\nu}$ where $c_{\nu}$ are any given numbers and $z=x+i y$ is a complex variable. To answer a question raised by the chemist Mendelieff, A. Markoff [3] proved the following theorem.

THEOREM A. If $\mathrm{p}(\mathrm{z})=\sum_{\nu=0}^{\mathrm{n}} \mathrm{c}_{v} \mathrm{z}^{v}$ is a polynomial of degree $\mathrm{n}$, and $|\mathrm{p}(\mathrm{x})| \leqq 1$ in the interval $-1 \leqq \mathrm{x} \leqq 1$ then in the same interval

$$
\left|p^{\prime}(x)\right| \leqq n^{2}
$$

The result is best possible, but for every subinterval of $[-1,1]$ the following result of Bernstein provides a much better estimate.

THEOREM B. Under the conditions of Theorem A

$$
\left|p^{\prime}(x)\right| \leqq n\left(1-x^{2}\right)^{-1 / 2} \quad(-1<x<1) .
$$

For real-valued polynomials having no roots in the interior of the unit circle, Erdös [2] proved the following sharper theorem.

Canad. Math. Bull. vol. 7, no. 1, January 1964 
THEOREM C. Let $\mathrm{p}(\mathrm{z})$ be a real-valued polynomial of degree $\mathrm{n}$ having no roots in the interior of the unit circle. If $|\mathrm{p}(\mathrm{x})| \leqq 1$ in the interval $-1 \leqq \mathrm{x} \leqq 1$ and $0<\mathrm{c}<1$, then for $-1+c<x<1-c$

$$
\left|p^{\prime}(x)\right|<\frac{4}{c^{2}} \sqrt{n}
$$

for $n>n_{0}$.

He also showed that in theorem $C, \sqrt{n}$ can not be replaced by any function tending to infinity more slowly.

We give an improvement on theorem $\mathrm{C}$, and prove the following.

THEOREM. Let $f(z)$ be a rational function which is the quotient of two polynomials of degrees $m$ and $n$ respectively. If $f(z)$ has neither zeros nor poles inside the unit circle and $|\mathrm{f}(\mathrm{x})| \leqq 1$ for $-1 \leqq \mathrm{x} \leqq 1$, then for $0<\mathrm{c}<1$ and $-1+c \leqq x \leqq 1-c$

$$
\left|f^{\prime}(x)\right|<\frac{[2(m+n)]^{1 / 2}}{c}
$$

and

$$
\left|f^{\prime \prime}(x)\right|<\frac{[2(m+n)]^{5 / 4}}{c^{2}},
$$

for $m>m_{0}$ and $n>n_{0}$.

Proof of the Theorem. Let $f(x)$ be positive for $-1<x<1$. Suppose that for a certain $x_{0}$ in $(-1+c, 1-c)$, $\left|f^{\prime}\left(x_{0}\right)\right|>\frac{[2(m+n)]^{1 / 2}}{c}$. Take $\left|x-x_{0}\right|<\frac{\sqrt{2} c}{\sqrt{m+n}} ;$ denote the roots of $\mathrm{p}(\mathrm{z})$ and $\mathrm{q}(\mathrm{z})$ by $\alpha_{1}, \alpha_{2}, \ldots, \alpha_{\mathrm{m}}$ and $\beta_{1}, \beta_{2}, \ldots, \beta_{\mathrm{n}}$ respectively. By hypothesis $\left|\alpha_{u}\right| \geqq 1$ for $1 \leqq v \leqq m$, and 
$\left|\beta_{\nu}\right| \geqq 1$ for $1 \leqq \nu \leqq n . \quad$ (We give a proof for $f^{\prime}\left(x_{0}\right)>0$;

the proof for $\mathrm{f}^{\prime}\left(\mathrm{x}_{0}\right)<0$ is similar.) Hence

(3) $\frac{f^{\prime}\left(x_{0}\right)}{f\left(x_{0}\right)}=\sum_{i=1}^{m} \frac{1}{x_{0}-\alpha_{i}}-\sum_{i=1}^{n} \frac{1}{x_{0}-\beta_{i}} \geqq \frac{[2(m+n)]^{1 / 2}}{c}$.

Divide the interval $\left(x_{0}, x_{0}+\frac{\sqrt{2} c}{\sqrt{m+n}}\right)$ into $k$ equal parts. Then for $x_{0}+\frac{\sqrt{2} c}{\sqrt{m+n}} \cdot \frac{\lambda-1}{k}<x<x_{0}+\frac{\sqrt{2} c}{\sqrt{m+n}} \cdot \frac{\lambda}{k}$, where $1 \leqq \lambda \leqq k$, we have

$$
\left|\frac{1}{x-\alpha_{i}}-\frac{1}{x_{0}-\alpha_{i}}\right|=\frac{\left|x-x_{0}\right|}{\left|x-\alpha_{i}\right|\left|x_{0}-\alpha_{i}\right|}<\frac{\sqrt{2}}{c \sqrt{m+n}} \cdot \frac{\lambda}{k}
$$

$$
\left|\frac{1}{x_{0}-\beta_{i}}-\frac{1}{x-\beta_{i}}\right|=\frac{\left|x-x_{0}\right|}{\left|x-\beta_{i}\right|\left|x_{0}-\beta_{i}\right|}<\frac{\sqrt{2}}{c \sqrt{m+n}} \cdot \frac{\lambda}{k}
$$

so that

$$
\left|\sum_{i=1}^{m}\left(\frac{1}{x-\alpha_{i}}-\frac{1}{x_{0}-\alpha_{i}}\right)+\sum_{i=1}^{n}\left(\frac{1}{x_{0}-\beta_{i}}-\frac{1}{x-\beta_{i}}\right)\right|<\frac{[2(m+n)]^{1 / 2}}{c} \cdot \frac{\lambda}{k}
$$

and

$$
\begin{aligned}
\frac{f^{\prime}(x)}{f(x)} & =\sum_{i=1}^{m} \frac{1}{x-\alpha_{i}}-\sum_{i=1}^{n} \frac{1}{x-\beta_{i}} \\
& =\frac{f^{\prime}\left(x_{0}\right)}{f\left(x_{0}\right)}+\sum_{i=1}^{m}\left(\frac{1}{x-\alpha_{i}}-\frac{1}{x_{0}-\alpha_{i}}\right)+\sum_{i=1}^{n}\left(\frac{1}{x_{0}-\beta_{i}}-\frac{1}{x-\beta_{i}}\right) \\
& >\frac{f^{\prime}\left(x_{0}\right)}{f\left(x_{0}\right)}-\frac{[2(m+n)]^{1 / 2}}{c} \cdot \frac{\lambda}{k}>\frac{[2(m+n)]^{1 / 2}}{c}\left(1-\frac{\lambda}{k}\right) .
\end{aligned}
$$

Hence $f^{\prime}(x)>f(x) \frac{[2(m+n)]^{1 / 2}}{c}\left(1-\frac{\lambda}{k}\right)$. 
Therefore $f(x)$ increases in this interval and $f(x)>f\left(x_{0}\right)$. Since $f^{\prime}\left(x_{0}\right)>\frac{[2(m+n)]^{1 / 2}}{c}$ by hypothesis, we have for $x_{0}+\frac{\sqrt{2} c}{\sqrt{m+n}} \cdot \frac{\lambda-1}{k}<x<x_{0}+\frac{\sqrt{2} c}{\sqrt{m+n}} \cdot \frac{\lambda}{k}$,

(7) $\quad f^{\prime}(x)>f(x) \frac{f^{\prime}\left(x_{0}\right)}{f\left(x_{0}\right)}-\frac{[2(m+n)]^{1 / 2}}{c} \cdot \frac{\lambda}{k}$

$$
\begin{aligned}
& >f^{\prime}\left(x_{0}\right)-\frac{[2(m+n)]^{1 / 2}}{c} \cdot \frac{\lambda}{k} \\
& >\frac{[2(m+n)]^{1 / 2}}{c}\left(1-\frac{\lambda}{k}\right) .
\end{aligned}
$$

But then,

$$
\begin{aligned}
1 & >f\left(x_{0}+\frac{\sqrt{2 c}}{\sqrt{m+n}}\right)-f\left(x_{0}\right) \\
& =\int_{x_{0}}^{y_{0}} f^{\prime}(x) d x \quad\left(y_{0}=x_{0}+\frac{\sqrt{2} c}{\sqrt{m+n}}\right) \\
& =\sum_{\lambda=1}^{k} \int_{1}^{y_{1} 2} f^{\prime}(x) d x \quad\left(y_{1}=x_{0}+\frac{\sqrt{2} c}{\sqrt{m+n}} \cdot \frac{\lambda-1}{k} ;\right. \\
& \sum_{\substack{\lambda=1 \\
\lambda=1}} \frac{\left.y_{2}=x_{0}+\frac{\sqrt{2} c}{\sqrt{m+n}} \cdot \frac{\lambda}{k}\right)}{k}\left(1-\frac{\lambda}{k}\right) \frac{\sqrt{2} c}{\sqrt{m+n}} \cdot \frac{1}{k} \\
& =\sum_{\lambda=1}^{\sum} 2\left(1-\frac{\lambda}{k}\right) \frac{1}{k} \rightarrow 1 \text { as } k \rightarrow \infty \cdot
\end{aligned}
$$

This contradiction proves (1). 
If $f(z)=\frac{p(z)}{q(z)}$ is typically real and all the conditions of our theorem are satisfied, then for $\varepsilon>0$ the function

$$
F(z)=1+\varepsilon-f(-z)=\frac{P(z)}{Q(z)}
$$

is the quotient of two polynomials $P(z)$ and $Q(z)$ of degrees $\max (m, n)=m$ (say) and $n$ respectively, $P(z)$ and $Q(z)$ do not have zeroes in the unit circle, and $|f(x)| \leqq 1+\varepsilon$ for $-1 \leqq x \leqq 1$. If $f(x)>0$ for $-1<x<1$, then $F(x)>0$ for $-1<x<1$. Besides, $F^{\prime}(x)=f^{\prime}(-x)$. Hence if $f^{\prime}\left(x_{0}\right) \geqq \frac{(1+\varepsilon) \sqrt{m+n}}{c}$, then $F^{\prime}\left(-x_{0}\right) \geqq \frac{\sqrt{m+n}}{c}$. As for $(7)$, it follows that for $-x_{0}+\frac{c}{\sqrt{m+n}} \cdot \frac{\lambda-1}{k}<x<-x_{0}+\frac{c}{\sqrt{m+n}} \cdot \frac{\lambda}{k}$,

$$
\left|F^{\prime}(x)\right|>\frac{(1+\varepsilon) \sqrt{m+n}}{c}\left(1-\frac{\lambda}{k}\right) \text {, }
$$

i. e. , $\quad\left|f^{\prime}(x)\right|>\frac{(1+\varepsilon) \sqrt{m+n}}{c}\left(1-\frac{\lambda}{k}\right)$

for $x_{0}-\frac{c}{\sqrt{m+n}} \cdot \frac{\lambda}{k}<x<x_{0}-\frac{c}{\sqrt{m+n}} \cdot \frac{\lambda-1}{k}$.

Also (see the proof $(7)) \quad\left|f^{\prime}(x)\right|>\frac{(1+\varepsilon) \sqrt{m+n}}{c}\left(1-\frac{\lambda}{k}\right)$ for $x_{0}+\frac{c}{\sqrt{m+n}} \cdot \frac{\lambda-1}{k}<x<x_{0}+\frac{c}{\sqrt{m+n}} \cdot \frac{\lambda}{k}$ and then

$$
\begin{gathered}
1>\int_{y_{3}}^{y^{4}} f^{\prime}(x) d x \quad\left(y_{3}=x_{0}-\frac{c}{\sqrt{m+n}} ; y_{4}=x_{0}+\frac{c}{\sqrt{m+n}}\right) \\
>2 \sum_{\lambda=1}^{k} \frac{(1+\varepsilon) \sqrt{m+n}}{c}\left(1-\frac{\lambda}{k}\right) \frac{c}{\sqrt{m+n}} \cdot \frac{1}{k} \rightarrow 1+\varepsilon \text { as } k \rightarrow \infty .
\end{gathered}
$$

Thus we get a contradiction.

Since $\varepsilon$ is arbitrary we can state the following corollary. If $f(z)$ is typically real and satisfies the conditions of the 
theorem, then for $-1+c<x<1-c$

$$
\left|f^{\prime}(x)\right|<\frac{\sqrt{m+n}}{c}
$$

provided $m \geqq n$.

Suppose now that for a certain $x_{0}$ in $(-1+c, 1-c)$, $f^{\prime \prime}\left(x_{0}\right) \geq \frac{[2(m+n)]^{5 / 4}}{c^{2}}$. Take $\left|x-x_{0}\right|<2^{1 / 4} c[(m+n)]^{-3 / 4}$, and let $f\left(x_{0}\right)>0, f^{\prime}\left(x_{0}\right)>0$. We give a proof for $f^{\prime \prime}\left(x_{0}\right)>0$; the proof for $f^{\prime \prime}\left(x_{0}\right)<0$ is similar. Now

(8)

$$
\begin{aligned}
& \frac{f^{\prime \prime}\left(x_{0}\right)}{f\left(x_{0}\right)}=\left\{\frac{f^{\prime}\left(x_{0}\right)}{f\left(x_{0}\right)}\right\}^{2}-\left[\frac{d}{d x}\left\{\frac{f^{\prime}(x)}{f(x)}\right\}\right]_{x=x_{0}} \\
& =\left\{\frac{f^{\prime}\left(x_{0}\right)}{f\left(x_{0}\right)}\right\}^{2}-\sum_{i=1}^{m} \frac{1}{\left(x_{0}-\alpha_{i}\right)^{2}}+\sum_{i=1}^{n} \frac{1}{\left(x_{0}-\alpha_{i}\right)^{2}} \\
& =\sum_{\mu \neq \nu} \frac{1}{\left(x_{0}-\alpha_{\mu}\right)\left(x_{0}-\alpha_{\nu}\right)}+\sum_{\mu \neq v} \frac{1}{\left(x_{0}-\beta_{\mu}\right)\left(x_{0}-\beta_{\nu}\right)} \\
& +2 \Sigma \frac{1}{\left(x_{0}-\beta_{i}\right)^{2}}-2 \Sigma \frac{1}{x_{0}-\alpha_{i}} \Sigma \frac{1}{x_{0}-\beta_{i}} \\
& \geqq \frac{[2(m+n)]^{5 / 4}}{c^{2}}
\end{aligned}
$$

Divide the interval $\left(x_{0}, x_{0}+2^{1 / 4} c(m+n)^{-3 / 4}\right)$ into $k$ equal parts. Then for $x_{0}+2^{1 / 4} c(m+n)^{-3 / 4} \frac{\lambda-1}{k}<x<x_{0}+2^{1 / 4} c(m+n)^{-3 / 4} \frac{\lambda}{k}$, where $1 \leqq \lambda \leqq k$ and $\mu \neq \nu$, we have 
(9)

$$
\begin{aligned}
& \left|\frac{1}{\left(\mathrm{x}-\alpha_{\mu}\right)\left(\mathrm{x}-\alpha_{\nu}\right)}-\frac{1}{\left(\mathrm{x}_{0}-\alpha_{\mu}\right)\left(\mathrm{x}_{0}-\alpha_{\nu}\right)}\right| \\
& \quad=\left|\frac{1}{\left(\mathrm{x}-\alpha_{\mu}\right)}\left[\frac{1}{\mathrm{x}-\alpha_{\nu}}-\frac{1}{\mathrm{x}_{0}-\alpha_{\nu}}\right]-\frac{1}{\left(\mathrm{x}_{0}-\alpha_{\nu}\right)}\left[\frac{1}{\mathrm{x}-\alpha_{\mu}}-\frac{1}{\mathrm{x}_{0}-\alpha_{\mu}}\right]\right| \\
& \quad<2^{5 / 4}(\mathrm{~m}+\mathrm{n})^{3 / 4} c^{2} \cdot \frac{\lambda}{\mathrm{k}},
\end{aligned}
$$

(10)

$$
\left|\frac{1}{\left(x-\beta_{\mu}\right)\left(x-\beta_{\nu}\right)}-\frac{1}{\left(x_{0}-\beta_{\mu}\right)\left(x_{0}-\beta_{\nu}\right)}\right|<2^{5 / 4}(m+n)^{3 / 4} c^{2} \cdot \frac{\lambda}{k}
$$

(11) $\left|\sum_{i=1}^{m} \frac{1}{x-\alpha_{i}} \sum_{i=1}^{n} \frac{1}{x-\beta_{i}}-\sum_{i=1}^{m} \frac{1}{x_{0}-\alpha_{i}} \sum_{i=1}^{n} \frac{1}{x_{0}-\beta_{i}}\right|$

$$
\begin{aligned}
& =\mid \sum_{i=1}^{m} \frac{1}{x-\alpha_{i}} \sum_{i=1}^{n}\left(\frac{1}{x-\beta_{i}}-\frac{1}{x_{0}-\beta_{i}}\right) \\
& \quad+\sum_{i=1}^{n} \frac{1}{x_{0}-\beta_{i}} \sum_{i=1}^{m}\left(\frac{1}{x-\alpha_{i}}-\frac{1}{x_{0}-\alpha_{i}}\right) \mid \\
& <2^{1 / 4}(m \cdot n+n \cdot m)(m+n)^{-3 / 4} c^{-2} \cdot \frac{\lambda}{k},
\end{aligned}
$$

(12)

$$
\begin{aligned}
\left.\right|_{i=1} ^{n}\left\{\frac{1}{\left(x-\beta_{i}\right)^{2}}-\frac{1}{\left(x_{0}-\beta_{i}\right)^{2}}\right\} \mid \\
\quad=\left|\sum_{i=1}^{n}\left[\frac{1}{x-\beta_{i}}-\frac{1}{x_{0}-\beta_{i}}\right] \cdot\left[\frac{1}{x_{0}-\beta_{i}}+\frac{1}{x-\beta_{i}}\right]\right| \\
<2^{5 / 4} n(m+n)^{-3 / 4} c^{-2} \cdot \frac{\lambda}{k} ;
\end{aligned}
$$

From (9), (10), (11) and (12) we deduce that 


$$
\begin{aligned}
\left|\frac{f^{\prime \prime}(\mathrm{x})}{f(\mathrm{x})}-\frac{f^{\prime \prime}\left(\mathrm{x}_{0}\right)}{f\left(\mathrm{x}_{0}\right)}\right|= & \mid \sum_{\mu \neq \nu} \sum\left(\frac{1}{\left(\mathrm{x}-\alpha_{\mu}\right)\left(\mathrm{x}-\alpha_{\nu}\right)}-\frac{1}{\left(\mathrm{x}_{0}-\alpha_{\mu}\right)\left(\mathrm{x}_{0}-\alpha_{\nu}\right)}\right) \\
& +\sum_{\mu \neq \nu}\left(\frac{1}{\left(\mathrm{x}-\beta_{\mu}\right)\left(\mathrm{x}-\beta_{\nu}\right)}-\frac{1}{\left(\mathrm{x}_{0}-\beta_{\mu}\right)\left(\mathrm{x}_{0}-\beta_{\nu}\right)}\right) \\
& +2\left\{\frac{1}{\left(\mathrm{x}-\beta_{\mathrm{i}}\right)^{2}}-\frac{1}{\left(\mathrm{x}_{0}-\beta_{\mathrm{i}}\right)^{2}}\right\} \\
& \left.-2 \sum \frac{1}{\mathrm{x}-\beta_{i}}\right\}^{\Sigma} \frac{1}{\mathrm{x}-\alpha_{i}}+\Sigma \frac{1}{\mathrm{x}_{0}-\alpha_{i}} \Sigma \frac{1}{\mathrm{x}_{0}-\beta_{i}} \\
& <\frac{[2(\mathrm{~m}+\mathrm{n})]^{5 / 4}}{\mathrm{c}} \cdot \frac{\lambda}{\mathrm{k}} \cdot
\end{aligned}
$$

It follows that

$$
\begin{gathered}
\frac{f^{\prime \prime}(x)}{f(x)}=\frac{f^{\prime \prime}\left(x_{0}\right)}{f\left(x_{0}\right)}+\left[\frac{f^{\prime \prime}(x)}{f(x)}-\frac{f^{\prime \prime}\left(x_{0}\right)}{f\left(x_{0}\right)}\right]>\frac{f^{\prime \prime}\left(x_{0}\right)}{f\left(x_{0}\right)}-\left|\frac{f^{\prime \prime}(x)}{f(x)}-\frac{f^{\prime \prime}\left(x_{0}\right)}{f\left(x_{0}\right)}\right| \\
>\frac{f^{\prime \prime}\left(x_{0}\right)}{f\left(x_{0}\right)}-\frac{[2(m+n)]^{5 / 4}}{c^{2}} \frac{\lambda}{k} .
\end{gathered}
$$

Hence

$$
f^{\prime \prime}(x)>f(x) \frac{f^{\prime \prime}\left(x_{0}\right)}{f\left(x_{0}\right)}-\frac{2(m+n)^{5 / 4}}{c^{2}} \cdot \frac{\lambda}{k} .
$$

For

$$
\mathrm{x}_{0}+2^{1 / 4} \mathrm{c}(\mathrm{m}+\mathrm{n})^{-3 / 4} \frac{\lambda-1}{\mathrm{k}}<\mathrm{x}<\mathrm{x}_{0}+2^{1 / 4} \mathrm{c}(\mathrm{m}+\mathrm{n})^{-3 / 4} \frac{\lambda}{\mathrm{k}},
$$

$f(x)$ increases and $f(x)>f\left(x_{0}\right)$. If $f^{\prime \prime}(x)>0$, this is obvious. If $f^{\prime \prime}(x)<0$ we can prove that $f^{\prime}(x)$ can never be negative in this interval. To prove this suppose $f^{\prime}(x)=0$ for a certain 
$\mathbf{x}$ in this interval. Then

$$
\begin{aligned}
f^{f^{\prime}\left(x_{0}\right)} \frac{f^{\prime}\left(x_{0}\right)}{f\left(x_{0}\right)} \mid & =\mid \frac{f^{\prime}(x)}{f\left(x_{0}\right)}-\frac{f(x)}{f(x)} \\
& =\left|\sum_{i=1}^{m}\left(\frac{1}{x_{0}-\alpha_{i}}-\frac{1}{x-\alpha_{i}}\right)+\sum_{i=1}^{n}\left(\frac{1}{x-\beta_{i}}-\frac{1}{x_{0}-\beta_{i}}\right)\right| \\
& <\frac{[2(m+n)]^{1 / 4}}{c},
\end{aligned}
$$

so that

$$
\left|f^{\prime}\left(x_{0}\right)\right|<\frac{[2(m+n)]^{1 / 4}}{c}
$$

On differentiating

$$
f^{\prime}(x)=f(x)\left[\sum_{i=1}^{m} \frac{1}{x-\alpha_{i}}-\sum_{i=1}^{n} \frac{1}{x-\beta_{i}}\right]
$$

with respect to $x$ and using (13), we have

$$
\left|f^{\prime \prime}\left(x_{0}\right)\right|<\frac{[2(m+n)]^{5 / 4}}{c^{2}} \text {. }
$$

This contradicts the hypothesis that $\left|f^{\prime \prime}\left(x_{0}\right)\right| \geqq \frac{[2(m+n)]^{5 / 4}}{c^{2}}$.

Therefore we have

$$
f^{\prime \prime}(x)>f^{\prime \prime}\left(x_{0}\right)-\frac{[2(m+n)]^{5 / 4}}{c^{2}}>\frac{[2(m+n)]^{5 / 4}}{c^{2}}\left(1-\frac{\lambda}{k}\right) \text {. }
$$

But then, 


$$
\begin{aligned}
& \frac{[2(m+n)]^{1 / 2}}{c}>f^{\prime}\left(x_{0}+2^{1 / 4} c(m+n)^{-3 / 4}\right)-f^{\prime}\left(x_{0}\right) \\
& =\int_{y_{5}}^{y_{6}} f^{\prime \prime}(x) d x \quad\left(y_{5}=x_{0} ; y_{6}=x_{0}+2^{1 / 4} c(m+n)^{-3 / 4}\right)
\end{aligned}
$$

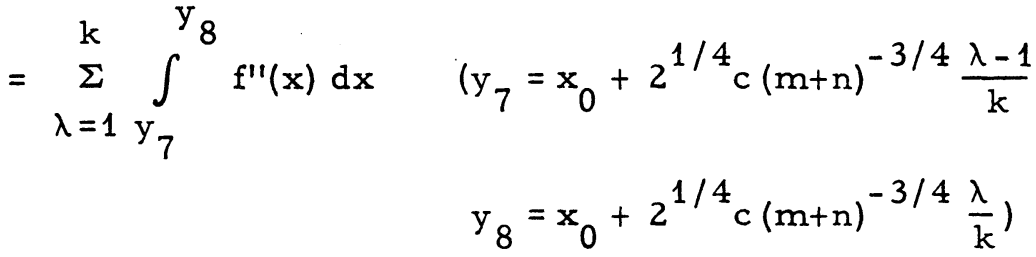

$$
\begin{aligned}
& >\sum_{\lambda=1}^{k} \frac{[2(m+n)]^{5 / 4}}{c^{2}}\left(1-\frac{\lambda}{k}\right) 2^{1 / 4} c(m+n)^{-3 / 4} \cdot \frac{1}{k} \\
& =\sum_{\lambda=1}^{k} \frac{2[2(m+n)]^{1 / 2}}{c}\left(1-\frac{\lambda}{k}\right) \cdot \frac{1}{k} \\
& \rightarrow \frac{[2(m+n)]^{1 / 2}}{c} \text { as } k \rightarrow \infty \text {. }
\end{aligned}
$$

This contradiction proves (2).

I thank Dr. Q. I. Rahman for suggesting this problem to me and assisting me in the preparation of this paper.

\section{REFERENCES}

1. S. Bernstein, Sur I'ordre de la meilleure approximation des fonctions continues par des polynomes de degré donné, Mémoires de I' Académie Royale de Belgique, (2), 4(1912), $1-103$.

2. P. Erdös, On extremal properties of the derivative of the polynomials, Annals of Math. 41 (1940), 310-313. 
3. A. Markoff, On a certain problem of D.I. Mendelieff, Utcheniya Zapiski Imperatorskoi Akademie Nauk.

(Russia) 62 (1889), 1-24.

Université de Montréal

and

Regional Engineering College, Srinagar, India 